Tag diversity of inkjet printed body-worn radio frequency identification integrated medical sticking plasters for wireless monitoring

Dumtoochukwu O. Oyeka, John C. Batchelor, Ali M. Ziai, Joseph Wheeler, Stephen Yeates

This paper is a postprint of a paper submitted to and accepted for publication in IET Microwaves, Antennas and Propagation and is subject to Institution of Engineering and Technology Copyright. The copy of record is available at IET Digital Library. 


\title{
Tag diversity of inkjet printed body-worn radio frequency identification integrated medical sticking plasters for wireless monitoring
}

\author{
Dumtoochukwu O. Oyeka', John C. Batchelor ${ }^{1}$, Ali M. Ziai ${ }^{1}$, Joseph Wheeler ${ }^{2}$, Stephen \\ Yeates $^{2}$
}

\author{
'School of Engineering and Digital Arts, University of Kent, Canterbury, Kent CT2 7NT, UK \\ 2Department of Chemistry, University of Manchester, Manchester M13 9PL, UK
}

E-mail: doo20@kent.ac.uk

Published in Healthcare Technology Letters; Received on 19th April 2016; Revised on 15th June 2016; Accepted on 16th June 2016

\begin{abstract}
This Letter presents an investigation of radio frequency identification tags integrated with sticking plasters. Conductive ink is used in the fabrication to provide good compatibility with the plaster in terms of flexibility. The tag is proposed for use as a wireless platform for long-term patient monitoring in hospital environments. A motion capture system is used to test the directions in which the tag can be read to inform a diversity study establishing the number of tags required on-body to ensure reliable reading for applications such as medical monitoring. Tag diversity performance with various body movements is also studied. It is established that four tags give all round body coverage with only $4 \%$ loss in coverage for vertically polarised tags should one of them fail.
\end{abstract}

1. Introduction: Radio frequency identification (RFID) has found use in areas such as access control systems, point of sale, automatic toll collection, animal tracking, vehicle tracking and immobilisation and also in the retail supply chain [1]. For medical applications, RFID technology can be used for patient tracking and safeguarding [2] as well as for equipment inventory management. RFID tags have also been used for physiological monitoring [3].

The use of wireless monitoring in a hospital environment aims to improve the efficiency of patient care. Advances have seen the use of RFID technology as a standalone system or in combination with other wireless technologies [4]. Proposed batteryless wireless health sensors [5] will transmit patient vital data and therefore the risks associated with signal outage are high making it important to keep skin mounted RFID tag read failure to a minimum.

Human tracking and monitoring can be achieved with RFID chips embedded in the skin [6], however, this is very short read range and is inappropriate for non-permanent applications. Alternatively, RFID wristbands [7] are widely used, but must go on the wrist or ankle and offer only a loose contact to the skin which compromises their use for monitoring. For instance, it may not be optimal for physiological measurements such as heartbeat and pulse which require close skin contact [8]. The use of RFID for medical sensing has also been reported in works such as [9] where an adhesive sensor badge is reported for sensing biomarkers in sweat and in [10] for monitoring surface temperature.

Diversity is a technique used in communication systems where alternative transmission mechanisms are introduced to reduce the chance of losing communication (signal outage). In RFID, the chances of successfully reading a tagged object or person are increased if more than one tag is used. This is referred to as tag diversity. In [11], a plaster operating over a few centimetres at the near field communication (NFC) frequency of $13.56 \mathrm{MHz}$ was presented and in [12] the most efficient tag placement for two worn ultra high frequency (UHF) tags communicating with an external reader was studied using $3 \mathrm{~mm}$ thick textile slotted patch antennas. The study indicated that more than two tags of the slotted patch design were required to provide coverage all around the body. Other works such as [13] have sought to improve the performance of on-body RFID tags by using dual tag antenna diversity to reduce the probability of phase cancellation between the RFID reader continuous carrier wave and the backscattered signal from the tag. Diversity is already often implemented at the reader end and in [14] a non-RFID body mounted wireless system utilises multiple antennas at the off-body base station. While this results in an improvement in outage, it will also lead to an increase in infrastructure cost due to the relatively expensive readers. Also, two antennas were used to implement selective combining diversity in [15] for on-body communication that was not RFID.

The work presented in this Letter assesses the performance of up to four $1.5 \mathrm{~mm}$ thick UHF tags that can be interrogated by a reader while avoiding read outage. The tag is integrated into a sticking plaster to ensure comfort and enable reading over extended periods. A multiple tag diversity study is carried out to establish tag configurations that result in uninterrupted read access. Such link reliability would be important for future tags performing a sensing function on the skin where there is a need to stream information. The implementation of the multiple tag system introduced in this Letter presents a low-cost alternative for using additional readers or complex signal processing algorithms which have been presented in previous work.

2. Sticking plaster RFID tag design: AHiggs-3 Electronic Product Code Class 1 Gen 2 RFID integrated circuit (IC) [16] with an impedance of 31-j216 $\Omega$ was matched to the tag antenna shown in Fig. 1. The sticking plaster integrated tag was derived from one created for mounting directly on the skin as a tattoo [17]. The antenna is a half wave dipole matched to the RFID transponder chip via a slot transformer. To ensure resilience to any detuning effect of the body, the tag was simulated using 
CST Microwave Studio® on a two layered block model of human tissue consisting of skin/fat and muscle using electrical parameters obtained from [18]. Tag efficiency is severely degraded on human tissue meaning the simulation was essential to ensure the tag was optimally matched to the transponder chip and achieve maximum read range. The highly porous sticking plaster substrate was represented by a $1.5 \mathrm{~mm}$ layer of empty space. The final tag had the dimensions given in Fig. 1 and operated at $867 \mathrm{MHz}$ with a fractional bandwidth of $11 \%$. This was sufficient to cover the European (867-868 MHz), USA (902-928 MHz) and Korean (905.5-914 MHz) UHF RFID bands.

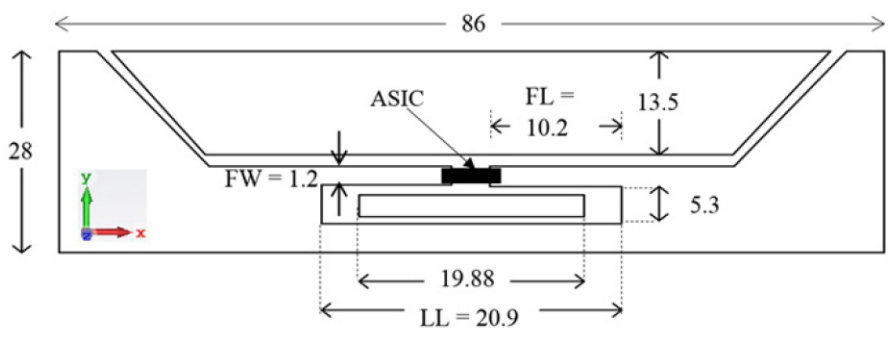

Fig. 1 Principal dimensions of the printed tag $(\mathrm{mm})$

The high permittivity of tissue means that tag radiation patterns are strongly affected by the body surface shape. Therefore, the tag was simulated on a torso created by three-dimensional (3D) scanning of the test subject. The co-polar elevation and azimuthal pattern cuts are shown in Figs. $2 \mathrm{a}$ and b, respectively, for the modelled torso in Fig. 2c. In free space, the tag has a dipole-like omni-directional radiation pattern, but when mounted on-body the pattern is directed forwards. A gain of $-12 \mathrm{dBi}$ was simulated due to compromised radiation efficiency caused by the thin tag as the antenna is separated from the skin by only $1.5 \mathrm{~mm}$. This corresponds to a read range of about $2 \mathrm{~m}$ for a moderate sensitivity chip (turn-on power $=-15 \mathrm{dBm}$ ) which is adequate for operation within a small room, or from bedside equipment.

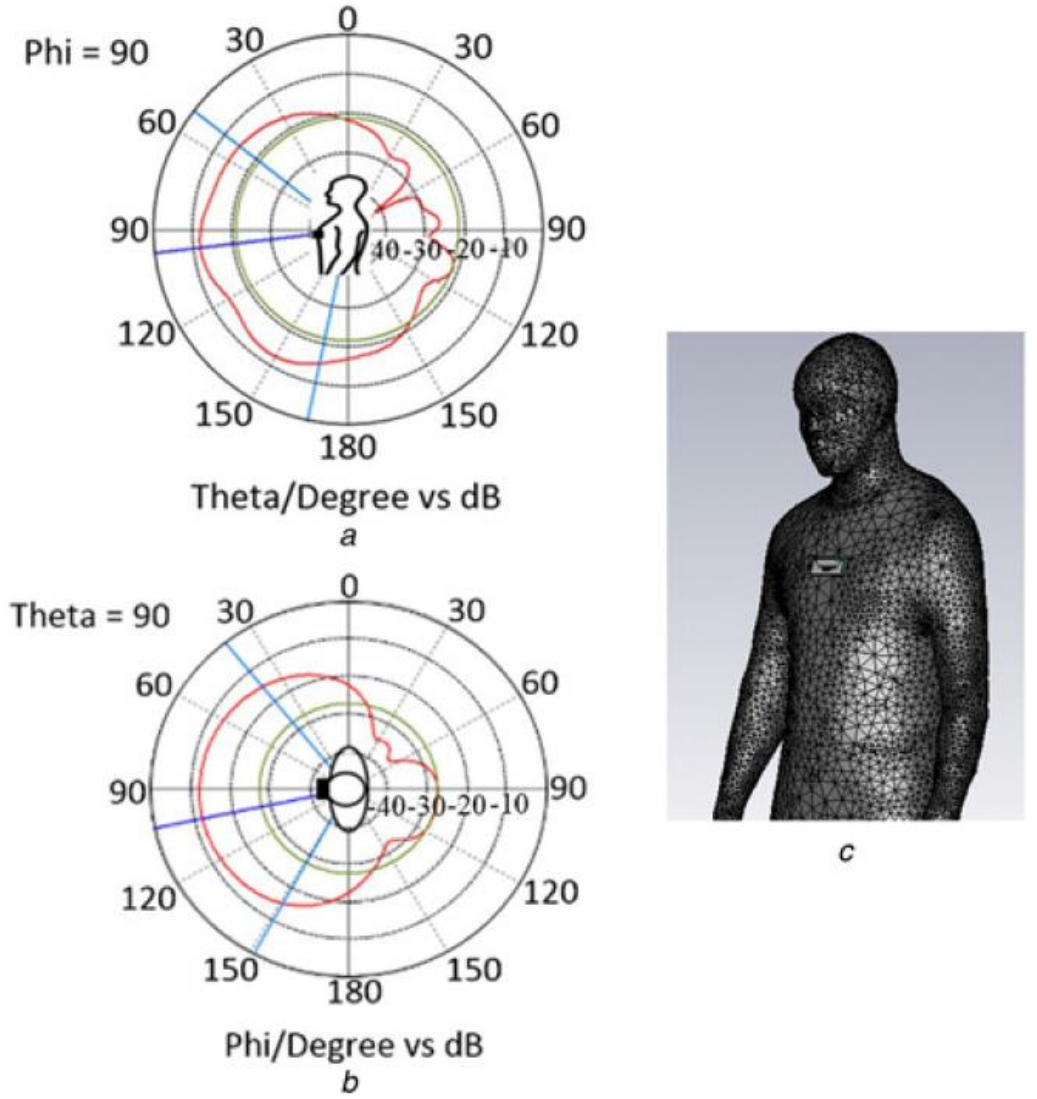

Fig. 2 Simulated co-polar radiation patterns at $867 \mathrm{MHz}$

$a$ E-component in elevation $(y-z)$ plane

$b$ E-component in azimuthal $(x-z)$ plane

$c$ Scanned 3D torso model

3. Inkjet printed on-body RFID tag: To be mechanically compatible with sticking plaster, the metalised tag antenna was fabricated with conductive ink using inkjet printing onto paper [19] to reduce skin irritation caused by extended wear. A Dimatix DMP-2800 inkjet printer [20] and silver nanoparticle ink by Sun Chemicals [21] were used. In order to achieve 
good definition and high electrical conductivity, the antennas were formed of two ink layers, with the second deposited after the initial layer had dried at room temperature. An ink dot spacing of $25 \mu \mathrm{m}$ was used. Finally, a Higgs-3 RFID application specific integrated circuit (ASIC) was attached to the tag with conductive glue and reinforced with an adhesive tape. The assembled tag is shown in Fig. 3.

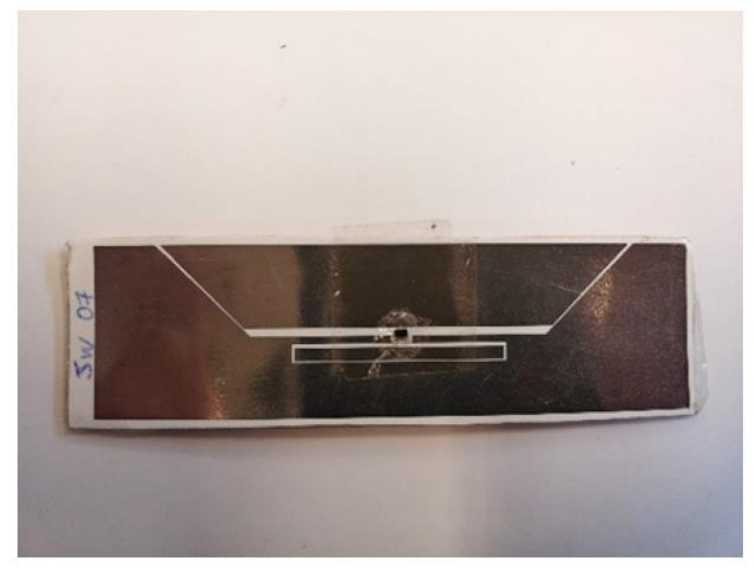

Fig. 3 Inkjet printed tag using conductive ink

4. On- and off-body tag measurements: Read range and performance measurements were first obtained using a Voyantic

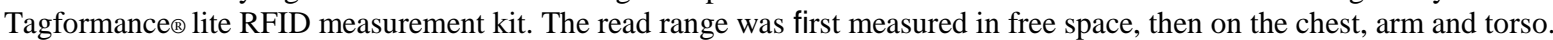
Fig. 4 shows that mounting the plaster on human tissue reduced the read range from about 3.75 to $2 \mathrm{~m}$ at $867 \mathrm{MHz}$, though when mounted on-skin the tag performance was relatively independent of frequency.

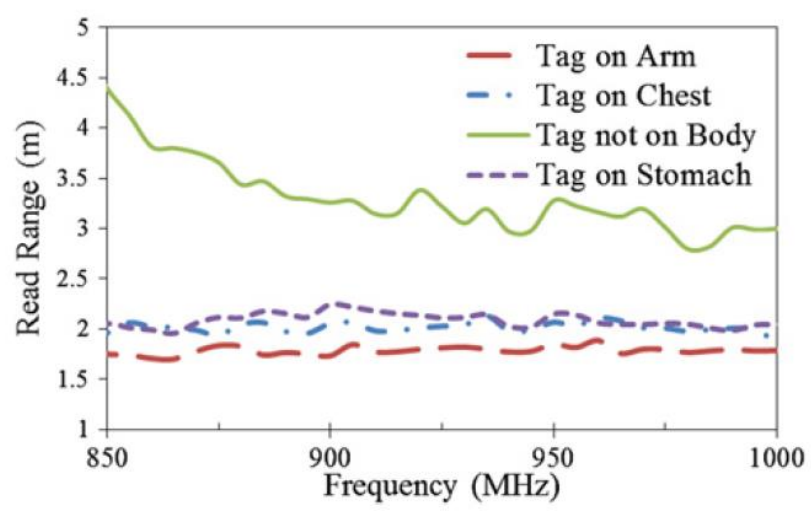

Fig. 4 Read range measurements for tag mounted on different parts of the body and isolated in air

Placing the tag on the abdomen or chest gave ranges of $2 \mathrm{~m}$, while mounting on the arm gave slightly lower values. The dependence of read range on body mounting location is attributed to the different composition of the cavity filled chest in comparison to the forearm which comprises denser muscle and bone. As the tag range was almost $2 \mathrm{~m}$, it was considered adequate for use in person monitoring applications inside buildings.

Tags used in future medical applications to stream critical signals will require diversity to reduce the risk of read outage. Therefore, a study was carried out using motion capture to establish the angles where no read occurred for a person wearing a number of tags. This was done for groups of two, three and four tags mounted on different body locations with the wearer turning on the spot.

5. Two-tag diversity: Two tags were each mounted on three volunteers (Fig. 5). The same human wearers were used for all measurements and the results were averaged over six consecutive identical measurements. A Vicon motion capture system [22] consisting of eight cameras was used to locate the tags and the circularly polarised reader antenna positions [23]. The coordinates of the markers were used to compute the relative angles between the tags and the read antenna. Measurements were taken in the same position of the $12 \mathrm{~m} \times 11 \mathrm{~m} \times 2.3 \mathrm{~m}$ height room. No absorbing material was used in order to represent an ordinary indoor environment.

It can be seen that in all two-tag cases (Figs. 5a-e), the body blockage and mounting effects have caused regions where neither tag read (grey sectors). 

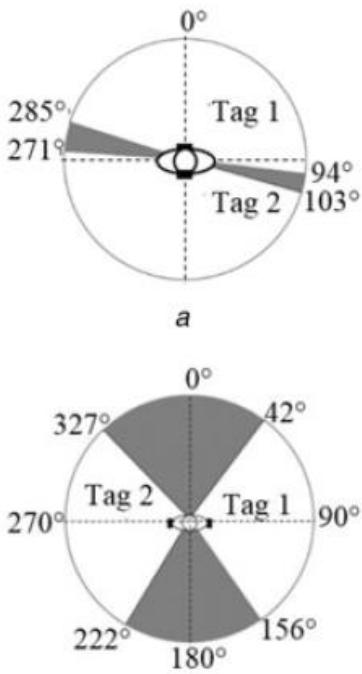

c

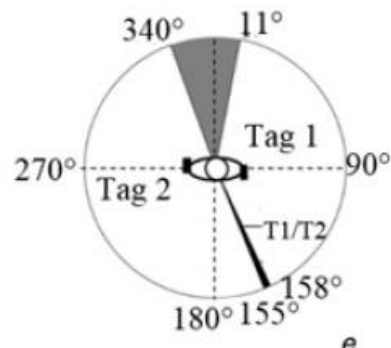

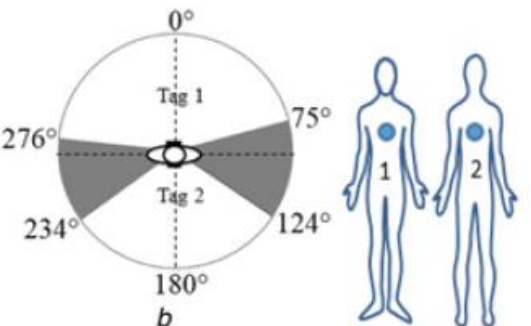

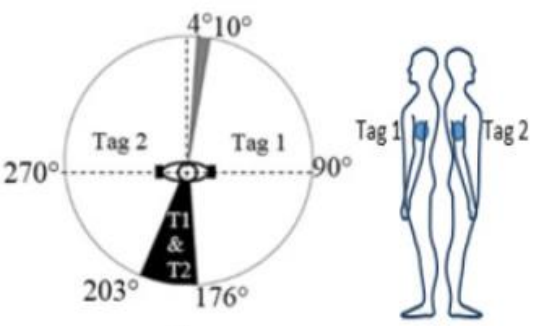

$d$

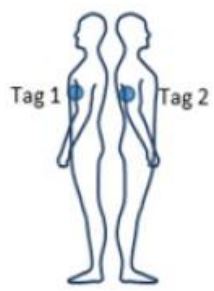

Fig. 5 Two-tag diversity study

$a$ Horizontal tags centred on chest and back

$b$ Vertical tags on chest and back

$c$ Horizontal tags mounted on upper arms

$d$ Vertical tags mounted symmetrically on upper arms

$e$ Vertical tags mounted asymmetrically on upper arms

On-body tag read sectors: white, black and grey are single tag, multiple tag

and no tag read sectors, respectively

Figs. 5a and b show the tags placed on the chest and back for horizontal and vertical tag orientations, respectively, where orientation refers to the tag longer side. There is better coverage for the horizontal tags with a total read coverage area of $337^{\circ}$ (94\% of area) compared with $270^{\circ}(75 \%)$ for the vertically oriented tags. When on the arms, the vertically oriented tags in Figs. 5c-e had wider coverage than the horizontal with 98 and $60 \%$ of the total area covered, respectively. In the vertical case, there was a region where the two tags read simultaneously as a result of the vertical tags experiencing lower body blockage. In a bid to increase the read coverage further, the vertical tags were placed asymmetrically on the arms (Fig 5e). However, this reduced the total read sector to $91 \%$ with an increase in the no-read region and a decrease in the overlapping sector.

In [12], it was noted that for $3 \mathrm{~mm}$ thick textile RFID body mounted tags, vertical polarisation offered better performance on the chest while horizontal tags were preferred on the arms. This is contrary to the data presented here, but is caused by difference in the antenna designs which had different polarisations.

It can be seen that in all the two-tag diversity cases, body blockage and mounting effects have caused regions where neither tag read (grey sectors). The best coverage was achieved for the two tags placed vertically on the arms with a total non-read width of just $6^{\circ}$. When the tags were placed horizontally, the non-read sector increased to $147^{\circ}$, the largest for any of the two-tag setups.

Additionally, Table 1 indicates a reduction in the coverage area should one of the two tags fail. With at most $48 \%$ outage, this failure has the least effect on the vertically placed tags on the arms compared to other 2 tag diversity system. As no combination of two tags offered complete round body coverage, three- and four-tag diversity was considered with the objective that for critical health applications a higher degree of diversity would be required.

6. Three-tag diversity: The tag locations for three-tag diversity were identified empirically to establish the configuration with the largest possible sectors of simultaneous reading. The presented results are an average of six consecutive measurements. 
Table 1 Outage sector widths for two-tag diversity on-body assuming one failed tag

\begin{tabular}{|c|c|c|c|}
\hline Tag setup & $\begin{array}{l}\text { Two-tag outage } \\
\text { region, } \%\end{array}$ & $\begin{array}{l}\text { Failed } \\
\text { tag }\end{array}$ & $\begin{array}{l}\text { Single-tag outage } \\
\text { region, } \%\end{array}$ \\
\hline chest and back $(\mathrm{H})$ & 6 & $\operatorname{tag} 1$ & 53 \\
\hline (Fig. $5 a$ ) & & $\operatorname{tag} 2$ & 53 \\
\hline chest and back (V) & 25 & $\operatorname{tag} 1$ & 69 \\
\hline (Fig. 5b) & & $\operatorname{tag} 2$ & 56 \\
\hline \multirow[t]{2}{*}{$\operatorname{arms}(\mathrm{H})($ Fig. $5 c)$} & 39 & $\operatorname{tag} 1$ & 71 \\
\hline & & $\operatorname{tag} 2$ & 68 \\
\hline \multirow[t]{2}{*}{ arms (V) (Fig. 5d) } & 2 & $\operatorname{tag} 1$ & 48 \\
\hline & & $\operatorname{tag} 2$ & 46 \\
\hline arms asymmetrical & 9 & $\operatorname{tag} 1$ & 49 \\
\hline (V) (Fig. 5e) & & $\operatorname{tag} 2$ & 59 \\
\hline
\end{tabular}

$\mathrm{H}$, horizontal polarisation and $\mathrm{V}$, vertical polarisation.

For three horizontal tags (Fig. 6a), 90\% coverage was obtained with regions of simultaneous multiple reads accounting for $19 \%$ of this coverage. There is a $36^{\circ}$ region of outage between tag 3 on the chest and tag 1 on the rear of the right arm. This represents $10 \%$ of the total area. The wide overlap region between tag 1 and 2 is expected since they are both rear facing.

For three vertical tags (Fig. 6b), the coverage gap between tag 1 and 3 was completely eliminated becoming a region of $2^{\circ}$ overlap. This is a significant improvement on the horizontally oriented tags.
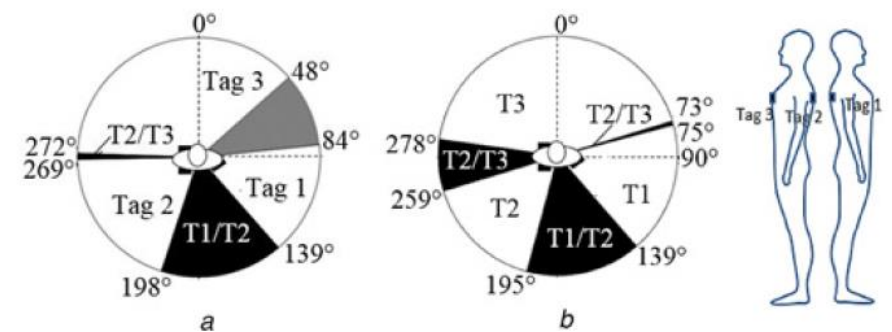

Fig. 6 Three-tag diversity study $a$ Horizontal tags

$b$ Vertical tags

White, black and grey sectors are single tag read, two-tag read and no-read sectors, respectively

Additionally, the regions of simultaneous tag reads increased to a total of $83^{\circ}$ which represents $23 \%$ of the total area and is an increase on the $62^{\circ}$ overlap region obtained with the horizontal tags. The increased region of overlap indicates that wider coverage will be maintained by the vertically polarised tags should one fail or be blocked, for instance on a recumbent wearer.

The above results again indicate that vertically oriented tags provide better coverage than horizontal. However, the overlap of only $2^{\circ}$ between tags 1 and 3 means that the diversity is not robust. To assess the three-tag diversity robustness against single tag failure, the effect of removing each tag individually on the total outage region is summarised in Table 2, where the outage regions for the horizontally placed tags include the original region of no coverage between tags 3 and 1 . The effect of a single failed or blocked tag is less detrimental in the case of vertically oriented tags and is a result of the wider read sectors in this orientation which increases the overlapping read sectors. As there was still significant outage when a single tag was obscured, a four-tag diversity system was studied.

7. Four-tag diversity: For four horizontal tags (Fig. 7a), omni-directional reading occurred and the combined width of the simultaneous multi-tag read sectors was $190^{\circ}(53 \%)$ of the read area. With the tags placed vertically (Fig. $7 \mathrm{~b}$ ), the overlapping regions where at least two tags read represented $73 \%\left(263^{\circ}\right)$ of the total possible coverage. In addition to the better simultaneous tag read coverage afforded by the use of vertically oriented tags, it can be seen that this orientation provides a region of $26^{\circ}$ where tag 2 , tag 3 and tag 4 read at the same time. This provides very good robustness against blockage or failure. This is again consistent with the vertically oriented tags providing better on-body performance than horizontally oriented tags. 
Table 2 Read sector widths for three-tag diversity on-body assuming one failed tag

\begin{tabular}{lccc}
\hline Tag setup & $\begin{array}{c}\text { Three-tag outage } \\
\text { region, \% }\end{array}$ & $\begin{array}{c}\text { Failed } \\
\text { tag }\end{array}$ & $\begin{array}{c}\text { Two-tag outage } \\
\text { region, \% }\end{array}$ \\
\hline $3(\mathrm{H})$ & 10 & $\operatorname{tag~1}$ & 25 \\
(Fig. $6 a)$ & & $\operatorname{tag} 2$ & 30 \\
& & $\operatorname{tag} 3$ & 48 \\
$3(\mathrm{~V})$ & 0 & $\operatorname{tag} 1$ & 18 \\
(Fig. $6 b)$ & & $\operatorname{tag} 2$ & 16 \\
& & tag 3 & 43 \\
\hline
\end{tabular}

$\mathrm{H}$, horizontal polarisation and $\mathrm{V}$, vertical polarisation.

Additionally, the regions of simultaneous tag reads increased to a total of $83^{\circ}$ which represents $23 \%$ of the total area and is an increase

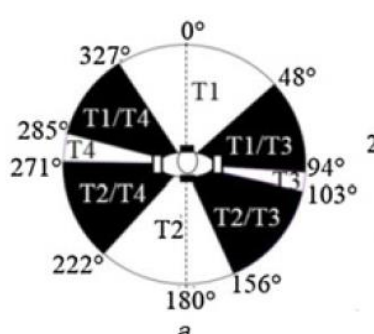

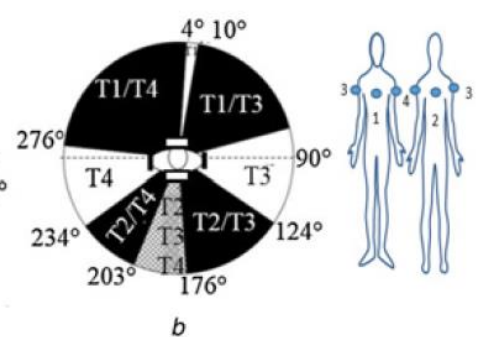

Fig. 7 Four-tag diversity study

$a$ Horizontal tags

$b$ Vertical tags

Gray, black and hashed are single tag and two-tag and three-tag simultaneous read sectors, respectively

\begin{tabular}{|c|c|c|c|}
\hline Tag setup & $\begin{array}{l}\text { Four-tag outage } \\
\text { region, } \%\end{array}$ & $\begin{array}{c}\text { Failed } \\
\text { tag }\end{array}$ & $\begin{array}{l}\text { Three-tag outage } \\
\text { region, } \%\end{array}$ \\
\hline $4(\mathrm{H})$ & 0 & $\operatorname{tag} 1$ & 23 \\
\hline \multirow[t]{3}{*}{ (Fig. 7a) } & & $\operatorname{tag} 2$ & 18 \\
\hline & & $\operatorname{tag} 3$ & 3 \\
\hline & & $\operatorname{tag} 4$ & 4 \\
\hline $4(\mathrm{~V})$ & 0 & $\operatorname{tag} 1$ & 2 \\
\hline \multirow[t]{3}{*}{ (Fig. 7b) } & & $\operatorname{tag} 2$ & 0 \\
\hline & & $\operatorname{tag} 3$ & 4 \\
\hline & & tag 4 & 2 \\
\hline
\end{tabular}

$\mathrm{H}$, horizontal polarisation and $\mathrm{V}$, vertical polarisation.

Table 4 Percentage read sector coverage for two-, three- and four-tag diversity on-body

\begin{tabular}{lccccc}
\hline & & \multicolumn{4}{c}{ Read sector coverage } \\
\cline { 3 - 6 } Study & Pol & Tag 1 & Tag 2 & Tag 3 & Tag 4 \\
\hline two tags & & chest & back & & \\
(chest-back) & $\mathrm{V}$ & $44 \%$ & $31 \%$ & - & - \\
& $\mathrm{H}$ & $47 \%$ & $47 \%$ & - & - \\
two tags & $\mathrm{V}$ & $54 \%$ & $52 \%$ & - & - \\
(arms) & $\mathrm{H}$ & $30 \%$ & $29 \%$ & - & - \\
three tags & & rear right arm & rear left arm & left chest & \\
& $\mathrm{V}$ & $41 \%$ & $38 \%$ & $51 \%$ & - \\
& $\mathrm{H}$ & $32 \%$ & $48 \%$ & $39 \%$ & - \\
four tags & & chest & back & right arm & left arm \\
& $\mathrm{V}$ & $44 \%$ & $31 \%$ & $54 \%$ & $53 \%$ \\
& $\mathrm{H}$ & $47 \%$ & $47 \%$ & $30 \%$ & $29 \%$ \\
\hline
\end{tabular}

The effect of the failure of any one of the tags on the overall coverage of the four-tag diversity system is summarised in Table 3. The results show very low percentage outage for the horizontal tags on the arms and is due to the wide read sector of the horizontal tags on the chest and back. The same explanation holds for the low outage percentage for tags 1 and 2 for the vertically oriented tags with tags 3 and 4 which have wide read sectors making up for the loss of any one of the tags. This indicates that a four-tag system is highly robust. Table 4 gives the percentage read sector widths for the two-, three- and four-tag diversity studies. Comparison shows some variation between the different tag diversities for both polarisations. 
This can be attributed to the differences in body motion of the individuals for the various measurements as well as possible minor variations in tag placement on each individual.

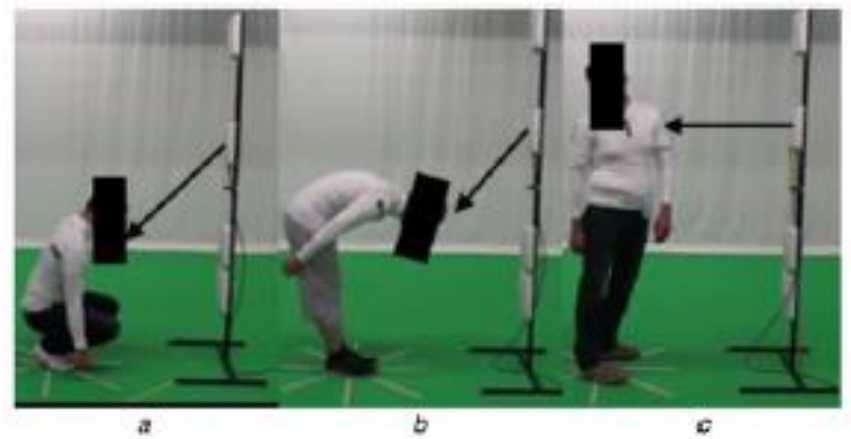

Fig 8 Different body motions

$a$ Picking

$b$ Bending

c Twising
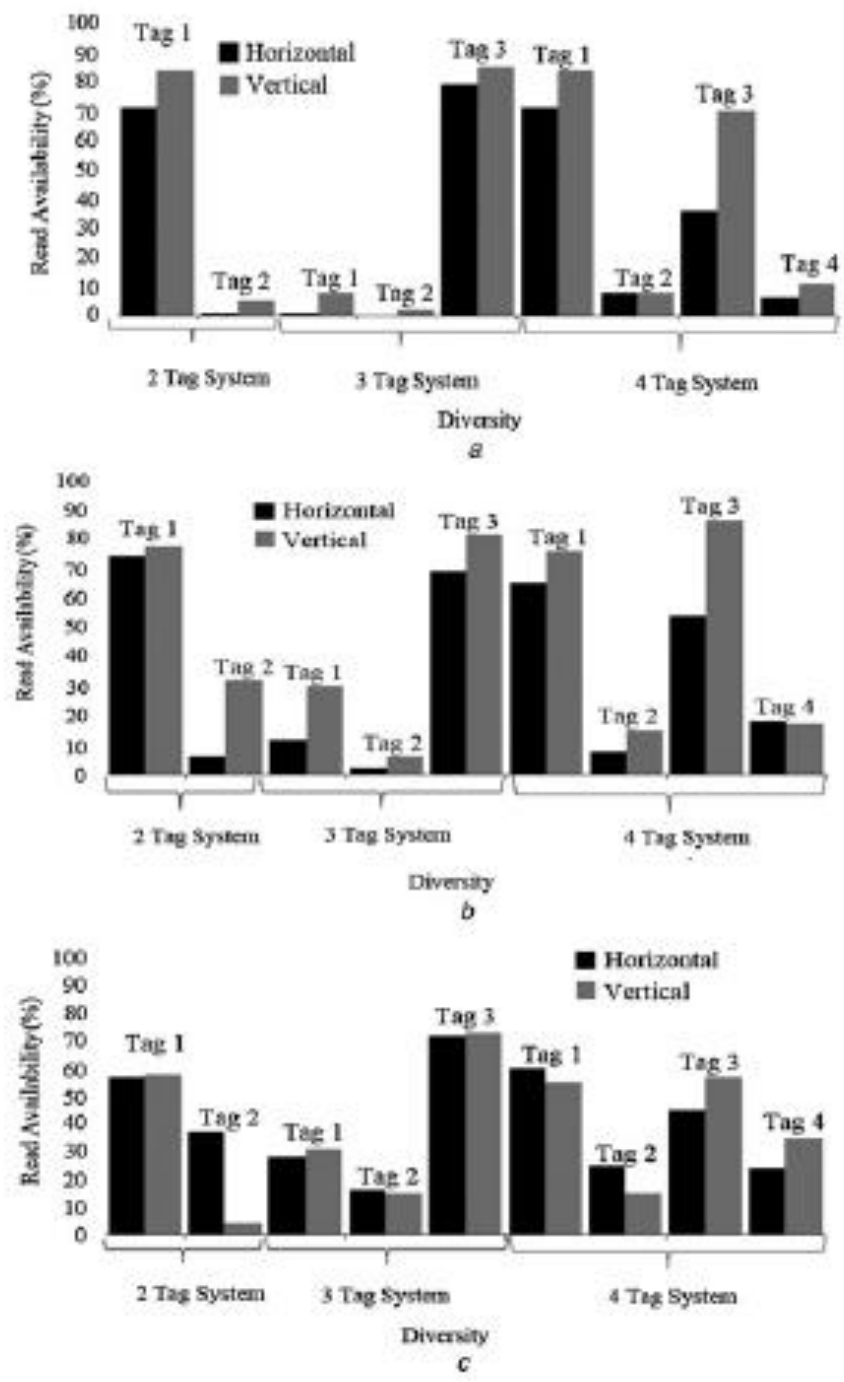

Fig 9 Percentage fag read for bedy motions

$a$ Crouching

$b$ Bending

$c$ Twisting 
We conclude that vertically mounted sticking plaster tags have better performance than horizontal in terms of combined read sector widths, though horizontal tags can also offer reliable omni-directional reading for four-tag diversity. All

measurements presented here refer to a single reader, and the use of multiple readers will improve read reliability and range at an increased infrastructure cost [12].

In Table 5, an overall performance rating of the two-, three- and four-tag diversities is given.

Table 5 Tag diversity performance assessment

\begin{tabular}{lcc}
\hline Tag setup & $\begin{array}{c}\text { Total } \\
\text { diversity }\end{array}$ & $\begin{array}{c}\text { Diversity with one failed } \\
\text { tag }\end{array}$ \\
\hline two tags chest/back (H) & good & poor \\
two tags chest/back (V) & moderate & poor \\
two tags arms (H) & good & poor \\
two tags arms (V) & poor \\
(symmetrical) & good & poor \\
two tags arms (V) & & \\
(asymmetrical) & good & poor \\
three tags $(\mathrm{H})$ & excellent & moderate \\
three tags $(\mathrm{V})$ & excellent & very good \\
four tags $(\mathrm{H})$ & excellent & excellent \\
four tags $(\mathrm{V})$ & &
\end{tabular}

8. Tag diversity performance with body movement: As a patient may be mobile while being monitored, the performance of multiple tags on the body during movement was studied. Two, three and four tags as defined in Sections 5-7 were placed on the body and the volunteers were asked to make crouching, bending and twisting motions. These motions were repeated for a fixed time and the number of tag reads was recorded for the reader which interrogates the tags about 35 times per second. A tag placed on the body while standing still for the same duration was used to obtain the maximum possible tag read count. Still photographs taken during the various continuous motions studied are shown in Figs. 8a-c.

A summary of the read availability is shown in Fig. 9 where again vertically orientated tags performed better than horizontal although the difference is marginal in some cases. Tags placed on the back had low read percentages due to body blocking as the user was facing forward, while tags on the chest also experienced some blocking during crouching and bending because of the position of the head during these motions. The tags on the three volunteers experienced varying degrees of blocking leading to outage because of the differences in their motion patterns as well as body shape.

9. Electromagnetic field safety: UHF RFID systems are licensed by regional regulation. A user $30 \mathrm{~cm}$ away from a reader antenna compliant with the EU permitted effective radiated power is exposed to a field strength below the allowed limit of $27.5 \mathrm{~V} / \mathrm{m}$. Therefore, correct installation of the reader antennas will ensure the system operates within the required safety limits.

10. Conclusion: Diversity studies of an inkjet printed sticking plaster RFID tag were carried out using motion capture equipment to ascertain which mounting positions offer reliable read coverage using four tags or fewer. Horizontally mounted tags on the chest and back provided better overall coverage than vertical tags which had narrower read sectors on the back. With wider read sectors, two vertical tags mounted symmetrically on the arms provided significantly better performance than the horizontally mounted tags. Three- and four-tag diversity offered improvement in read sector coverage. In the case of four-tag diversity, with tags on the arms and the chest and back, at least two tags read simultaneously in all overlap regions. The vertically oriented tags had overlap regions representing $73 \%$ of the coverage area compared with $53 \%$ for horizontally polarised tags. Four vertically oriented tags had a $26^{\circ}$ region where three tags read simultaneously. In this situation, reading should remain reliable even for recumbent patients on their backs or sides. Different body motions were also tested with read percentages again indicating marginally better overall performance for vertical tags. The studies inform the use of future passive skin mounted sensing and smart medical dressing applications in critical situations where signal outage must be minimised.

11. Funding and Declaration of Interests: This work and Mr Oyeka's studentship are funded by the UK Engineering and Physical Science Research Council (EPSRC, EP/J000825/1).

Conflict of interest: None declared.

12 References

[1] Secureidnews: 'Mexican firm offers RFID implants for kidnapping prevention - SecureIDNews'. Available at http://www.secureidnews. com/news-item/*mexican-firm-offers-rfid-implants-for-kidnappingprevention, accessed 04 August 2015 
[2] Ajami S., Rajabzadeh A.: 'Radio frequency identification (RFID) technology and patient safety', J. Res. Med. Sci., 2013, 32, (9), pp. 809-813

[3] Vaz A., Ubarretxena A., Zalbide I., ET AL.: 'Full passive UHF tag with a temperature sensor suitable for human body temperature monitoring', IEEE Trans. Circuits Syst. II, Express Briefs, 2010, 57, (2), pp. 95-99

[4] Catarinucci L., De Donno D., Mainetti L., ET AL.: 'Integration of UHF RFID and WSN technologies in healthcare systems'. RFID Technology and Applications Conf. (RFID-TA), Tampere, Finland, 2014, pp. 289-294

[5] Opasjumruskit K., Thanthipwan T., Sathusen O., ET AL.: 'Self-powered wireless temperature sensors exploit RFID technology',

IEEE Pervasive Comput., 2006, 5, (1), pp. 54-61

[6] Sirait D.C., Basari Zulkifli F.Y., Rahardjo E.T.: 'An implanted dipole antenna for RFID-based patient monitoring system'. Int. Conf. on QiR (Quality in Research), 2013, pp. 142-145

[7] Lopez-Soriano S., Parron J.: 'Wearable RFID tag antenna for healthcare applications'. IEEE-APS Topical Conf. on Antennas and Propagation in Wireless Communications (APWC), 2015, pp. 287-290

[8] Batchelor J.C., Casson A.J.: 'Inkjet printed ECG electrodes for long term biosignal monitoring in personalized and ubiquitous healthcare'. 37th Annual Int. Conf. of the IEEE Engineering in Medicine and Biology Society (EMBC), 2015, pp. 4013-4016 [9] Rose D.P., Ratterman M., Griffin D.K., ET AL.: 'System-level design of an RFID sweat electrolyte sensor patch'. 36th Annual Int. Conf. of the IEEE Engineering in Medicine and Biology Society, 2014, pp. 4038-4041

[10] Chen Y.C., Sun H.M., Chen R.S.: 'Design and implementation of wearable RFID tag for real-time ubiquitous medical care'. IEEE

Topical Conf. on Biomedical Wireless Technologies, Networks, and Sensing Systems (BioWireleSS), 2014, pp. 25-27

[11] Kollegger C., Steffan C., Greiner P., ET AL.: 'Intelligent plaster for accurate body temperature monitoring and investigations regarding EMI using near-field magnetic scan', Elektrotech. Inf.tech., 2016, 1, (33), pp. 25-31

[12] Manzari S., Occhiuzzi C., Marrocco G.: 'Feasibility of body-centric systems using passive textile RFID tags', IEEE Antennas Propag. Mag., 2012, 54, (4), pp. 49-62

[13] Wang J., Bolic M.: 'Exploiting dual-antenna diversity for phase cancellation in augmented RFID system'. Int. Conf. on Smart Communications in Network Technologies (SaCoNeT), 2014, pp. 1-6

[14] Yoo S.K., Cotton S.L.: 'Improving signal reliability for indoor off-body communications using spatial diversity at the base station'. The 8th European Conf. on Antennas and Propagation (EuCAP 2014), 2014, pp. 857-861

[15] Al-Saffar D., Habeeb E., Edwards R.M., ET AL.: 'Human effect on on-body selective combining at 2.4 GHz'. Antennas and Propagation Conf. (LAPC), Loughborough, United Kingdom, 2014, pp. 381-384

[16] Alien Technology: 'Higgs-3 product overview'. Available at http://www.alientechnology.com/docs/products/DS_H3.pdf, accessed 23 August 2011

[17] Ziai M., Batchelor J.C. : 'UHF RFID tag antenna design for on-body applications'. Proc. of Antennas and Propagation Conf. (LAPC), Loughborough, United Kingdom, 2010, pp. 185-188

[18] Ziai M.A., Batchelor J.C.: 'A prototype passive UHF RFID transfer tattoo tag'. Proc. of the 5th European Conf. on Antennas and Propagation, EUCAP. Rome, Italy, 2011, pp. 3811-3814

[19] Printed Electronics: 'PEL Nano-P60 paper'. Available at http://www.printedelectronics.co.uk/Information Sheet\%, accessed 01 December 2015

[20] Dimatix-Fujifilm Inc.: 'Dimatix presents DMP-2800 - a revolutionary materials deposition system'. Available at http://www. fujifilmusa.com/press/news/display_news?newsID=880140, accessed 19 January 2015

[21] Sun Chemicals: 'Silver nanoparticle ink'. Available at http://www.sigmaaldrich.com/catalog/product/aldrich/719048?, accessed 19 December 2014

[22] Vicon: 'What is motion capture'. Available at http://www.vicon.com/what-is-motion-capture, accessed 18 September 2015

[23] Swaisaenyakorn S., Young P.R., Batchelor J.C.: 'Animated human movement and posture capture for body worn antenna simulation'.

Proc. of the 5th European Conf. on Antennas and Propagation (EUCAP), 2011, pp. 3635-3639 